\title{
Perceptual Considerations for Quality of Service Management: An Integrated Architecture
}

\author{
George Ghinea and George D. Magoulas \\ Department of Information Systems and Computing, Brunel University, \\ Uxbridge, UB8 3PH, United Kingdom \\ \{George.Ghinea, George.Magoulas\}@brunel.ac.uk
}

\begin{abstract}
In this paper, we suggest an integrated architecture that makes use of the objective-technical information provided by the designer and the subjectiveperceptual information supplied by the user for intelligent decision making in the construction of communication protocols. Thus, this approach, based on the Analytic Hierarchy Process, incorporates not only classical Quality of Service (QoS) considerations, but, indeed, user preferences as well. Furthermore, in keeping with the task-dependent nature consistently identified in multimedia scenarios, the suggested communication protocols also take into account the type of multimedia application, which they are transporting. Lastly, our approach also opens the possibility for such protocols to dynamically adapt based on a changing operating environment.
\end{abstract}

\section{Introduction}

The focus of our research has been the enhancement of the traditional view of QoS with a user-level defined Quality of Perception (QoP). This is a measure which encompasses not only a user's satisfaction with multimedia clips, but also his/her ability to perceive, synthesise and analyse the informational content of such presentations. As such, we have investigated the interaction between QoP and QoS and its implications from both a user perspective [2] as well as a networking angle within the Dynamically Reconfigurable Stacks Project (DRoPS) [3]. In this work we address the problem of bridging the application-network gap from a multi-attribute decision making perspective. We have sought to use this approach to integrate results from our work on user-level Quality of Perception with the more technical characterisation of Quality of Service. Our ultimate aim is to provide a communications architecture which uses an adaptable communications protocol geared towards human requirements in the delivery of distributed multimedia.

\section{User-centred Design with Multi-criteria Constraints}

In linking perceptual considerations with low-level technical parameters, the design process should take into account the subjective judgement of the end-user. To this end, 
we have applied Saaty's AHP formalism [4] to obtain a method which, from combined user-, application- and network-level requirements, ultimately results in a protocol configuration specifically tailored for the respective user-needs. Thus, within the QoP framework, each multimedia application can be characterised by the relative importance of the video $(V)$, audio $(A)$ and textual $(T)$ components as conveyors of information, as well as the dynamism $(D)$ of the presentation. This agrees with the experimental QoP results obtained which emphasise that multimedia QoP varies with: the number of media flows, the type of medium, the type of application, and the relative importance of each medium in the context of the application. On the other hand, 5 network level QoS parameters have been considered: bit error $(B E R)$, segment loss $(S L)$, segment order $(S O)$, delay $(D E L)$ and jitter (JIT). Together with the $V, A, T$ and $D$ parameters these constitute the criteria on the basis of which an appropriate tailored communication protocol is constructed. In DRoPS, the functionality of this protocol is realised through a number of 9 microprotocols, which perform arbitrary protocol processing operations, spanning 4 broad functionality classes [3].

By applying the AHP we obtain a total of 10 matrices. The decision-maker (both the designer and the user) has to express his/her opinion about the value of a single pairwise comparison at a time. 9 of these matrices give the relative importance of the various microprotocols (alternatives, in the AHP) with respect to the criteria identified in our model, while the last of these matrices details pairwise comparisons between the criteria themselves. Thus, the judgement "microprotocol $A$ is equally important as microprotocol $B$ with respect to $B E R$ " corresponds to an entry $a_{i j}=1$ of a matrix,

while the judgement "microprotocol $A$ is absolutely more important than microprotocol $B "$ would correspond to a value of $a_{i j}=9$ [4]. Intermediate terms can also be assigned when compromise is needed between two adjacent characterisations. The tenth matrix, of each criterion with respect to all the other criteria, is the only one whose values may fluctuate as a result of changes in the operating environment, as well as a consequence of changes in user preferences and perceptions, and could conceptually be split-up into 4 sub-matrices (see Figure 1).

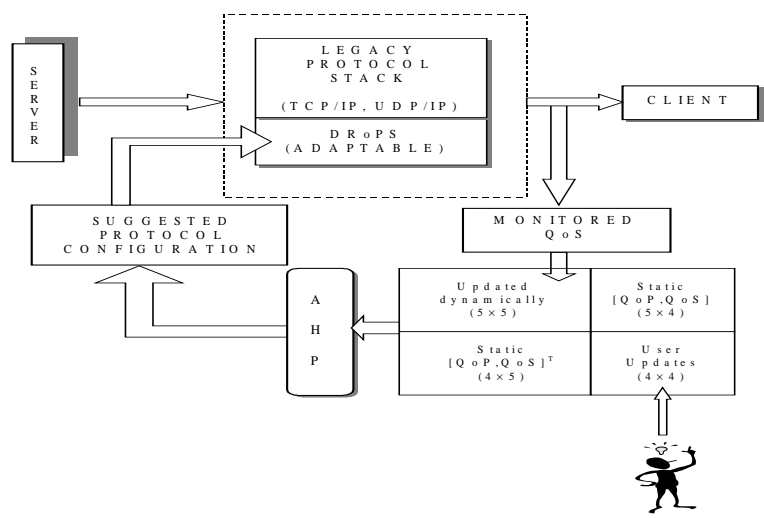

Fig. 1. AHP-based architecture for QoP management 
Following the AHP, the weights $w_{i}, i=1, \ldots 9$ denoting the relative importance of each criterion $i$ among the $p$ criteria ( $p=9)$ are evaluated using the formula:

$$
w_{i}=\frac{\left(\prod_{j=1}^{p} a_{i j}\right)^{1 / p}}{\sum_{i=1}^{p}\left(\prod_{j=1}^{p} a_{i j}\right)^{1 / p}} i=1,2, \ldots, p
$$

and a higher priority setting corresponds to a greater importance.

Pairs among alternatives are also compared with respect to the $i$ th criterion and then a weight $w_{j, i}$, which denotes how preferable is the alternative $j$ with respect to the criterion $i$, is derived. As previously, there is a total of $p$ pairwise comparisons in the matrix and weights are calculated following Relation (1). The weighted product model, [1], is used to compare alternative $v$ with alternative $u$. This can be done by multiplying a number of ratios, one for each criterion. Each ratio is raised to a power, which is equivalent to the relative weight of the corresponding criterion, i.e.

$$
P_{v / u}=\prod_{i=1}^{p}\left(\frac{w_{v, i}}{w_{u, i}}\right)^{w_{i}}
$$

If the ratio $P_{v / u}$ is greater or equal to one then the conclusion is that the alternative $v$ is more preferable than alternative $u$. In the maximisation case, the best alternative is the one that possesses the highest value among all others.

The applicability of the proposed approach can be illustrated by means of a multimedia user scenario tested through simulation: a currently executing BERsensitive application suddenly experiences deterioration in BER. Then this parameter becomes absolutely important with respect to the others i.e., $a_{l j}=9$ for $j=2, \ldots, 5$. This value is dynamically updated in the top-left $5 \times 5$ matrix (see Figure 1 ). The result of the AHP (calculations are omitted due to space limitation) then shows that the microprotocol that should preferentially be managed under the circumstances is the full Cyclic Redundancy Check - the strongest such microprotocol available to correct the quality loss in BER. This is in contrast to the scenario applicable just before the sudden surge in BER, which gave preference to an alternative microprotocol, the Strong Sequence Control.

\section{References}

1. Chen S.J. \& Hwang C.L.: Fuzzy multiple decision making, Lecture Notes in Economics and Mathematical Sciences, Vol. 375, NY, Springer, 1992.

2. Ghinea G. \& Thomas J.P.: QoS Impact on User Perception and Understanding of Multimedia Video Clips, Proc. of ACM Multimedia '98, Bristol, UK, 1998.

3. Ghinea G., Thomas J.P., Fish R.S.: Quality of Perception to Quality of Service Mapping Using a Dynamically Reconfigurable Communication System, Proc. of IEEE Globecom '99, Rio de Janeiro, Brazil

4. Saaty T.: The Analytic Hierarchy Process, McGraw-Hill, New York (1980). 